\title{
Caracterización de huertos urbanos y periurbanos de Xalapa de Enríquez, Veracruz
}

\author{
Characterization of urban and peri-urban gardens in Xalapa de Enríquez, Veracruz
}

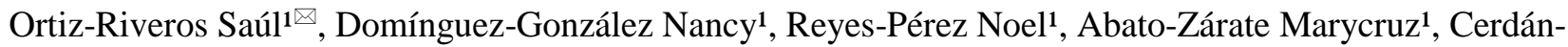 Cabrera Carlos R. ${ }^{1}$, Escalona-Aguilar Miguel A. ${ }^{1}$, Lozada García J. Armando ${ }^{2}$}

${ }^{1}$ Facultad de Ciencias Agrícolas Xalapa. Universidad Veracruzana. Circuito Universitario Gonzalo Aguirre Beltrán s/n. Col. Zona Universitaria. Xalapa. Veracruz. México. C. P. 91000.

${ }^{2}$ Facultad de Biología Xalapa. Universidad Veracruzana. Circuito Universitario Gonzalo Aguirre Beltrán s/n. Col. Zona Universitaria. Xalapa. Veracruz. México. C. P. 91000.

${ }^{凶}$ Autor para correspondencia: $\underline{\text { riveros922@gmail.com }}$

\section{RESUMEN}

La necesidad de adquirir alimentos de forma adecuada y sana ha llevado a buscar y fortalecer alternativas para su producción; en las ciudades se practica la agricultura urbana y periurbana (AUyP) y Xalapa, Veracruz, México, es un ejemplo de ello. Desde el año 2013 se autogestiono la Red de Agricultura Urbana y Periurbana de Xalapa (RAUPX), en la cual se centró el objetivo de la presente investigación, que consistió en caracterizar los huertos de la RAUPX, en función de las variables: tamaño de huerto, diversidad (índice de Shannon), presencia de artropofauna y prácticas de manejo fitosanitarias; con el fin de diagnosticar y aportar propuestas para mejorar el manejo integrado de plagas. Los resultados incluyen datos generales productor-huerto tales como rango de edad, nivel de estudios, superficie, familias botánicas representativas, presencia de artropofauna en relación con las especies cultivadas y manejo de prácticas fitosanitarias de acuerdo con su complejidad. Las prácticas fitosanitarias que realizan los productores son de forma correctiva en vez de preventiva y están moderadamente relacionadas con la presencia de plagas.

Palabras clave: agricultura urbana, biodiversidad, plagas, prácticas fitosanitarias, soberanía y seguridad alimentaria.

\begin{abstract}
The need to acquire food in an adequate and healthy way has led to seek and strengthen alternatives for their production; urban and peri-urban agriculture is practiced in the cities (AUyP) and Xalapa, Veracruz, Mexico, is an example of this. Since 2013, the Urban and Peri-urban Agriculture Network of Xalapa (RAUPX) has been self-managed, which focused on the objective of this research, which consisted in characterizing the gardens of the RAUPX, based on the variables: garden size, diversity (Shannon index), presence of arthropofauna and phytosanitary management practices, in order to diagnose and provide proposals for the improvement of integrated pest management. The results include general producergarden data such as age range, level of studies, area, representative botanical families, presence of artropofauna in relation to the cultivated species and management of phytosanitary practices according


to their complexity. Phytosanitary practices carried out by producers are corrective rather than preventive and are moderately related to the presence of pests.

Keywords: urban agriculture, biodiversity, pests, phytosanitary practices, sovereignty and food security.

\section{INTRODUCCIÓN}

La necesidad diaria de adquirir alimentos de forma justa y sana ha llevado a buscar y fortalecer alternativas de producción en las ciudades. A esto se le conoce como agricultura urbana y periurbana (AUyP) (Altieri et al., 2000), la cual ha prosperado en diversas partes del mundo. En el caso de México, se busca mejorar la alimentación de las familias y promover mejor calidad de vida de la población mediante la seguridad alimentaria (Toledo, 2002), por lo que esta forma de agricultura ha cobrado auge. En el caso de Xalapa, Veracruz, desde el 2013 se autogestiona una red cuyo propósito es intercambiar experiencias sobre la producción urbana de alimentos. La RAUPXcultiva en azoteas o terrazas, balcones, jardines particulares o privados, de uso común o público (Escalona et al., 2016). En este contexto, el objetivo fue caracterizar huertos urbanos y periurbanos de Xalapa, Veracruz de acuerdo con la superficie de sistema de producción, biodiversidad y prácticas de manejo fitosanitario.

\section{MATERIALES Y MÉTODOS}

Con base en los registros de 2014 de la Red de Agricultura Urbana y Periurbana de Xalapa, Veracruz, que en 2014 contaba con 30 ciudadanos con huertos. Participaron propietarios de 28 huertos que fueron visitados entre noviembre de 2015 y septiembre de 2016. Se registraron datos del propietario, así como el sexo, grado estudios, ocupación laboral. Con respeto al huerto se tomaron datos del tamaño (metros cuadrados), uso de la bitácora de trabajo, siembra, diversidad de especies medicinales, condimentarias y hortalizas, actividades fitosanitarias, tipo de huerto blando (siembra directa en suelo), duro (contenedores), mixto (ambos tipos), si era urbano o periurbano, presencia de plagas, y prácticas fitosanitarias. Adicionalmente, se les preguntó acerca del concepto de agricultura urbana y plaga; para ello se empleó el método de evaluaciones sumarias (escala Likert: siendo 1 el valor mínimo y 5 el máximo) para conocer la percepción de los agricultores sobre estos temas (Verdugo y Sabeh, 2002). Los datos obtenidos fueron organizados en una matriz, donde se categorizaron para facilitar su agrupación y posterior análisis.

Por otra parte, se analizó el número de plagas, tamaño de huerto, índice de diversidad de Shannon-Wiener y prácticas fitosanitarias; para identificar frecuencias, medidas de tendencia central y distribución de datos se utilizó el programa SPSS v.20.

\section{RESULTADOS Y DISCUSIÓN}

En la tabla 1 se muestra que el rango de edad de los productores osciló entre 20-70 años, el $50 \%$ de la población contaba con una edad de entre 20 y 40 años; la mayoría fueron mujeres $(72.6 \%)$. De acuerdo con Rivera (2006) es posible que el rango más activo que fue el de adultos jóvenes, sea el más participativo debido a la preocupación 
y conciencia en mejorar sus hábitos alimenticios cotidianos. Con respecto al grado de estudios, se observó que el $68 \%$ cuenta con estudios a nivel superior.

Tabla 1. Datos sociodemográficos de los productores de la RAUPX.

\begin{tabular}{cccc}
\hline Edades & Hombre & Mujer & Grado de estudios \\
\hline $20-30$ & $14.2 \%$ & $10.8 \%$ & $14 \%=$ Secundaria \\
$31-40$ & $3 \%$ & $22 \%$ & $18 \%=$ Preparatoria \\
$41-50$ & $7 \%$ & $7 \%$ & $60 \%=$ Licenciatura \\
$51-60$ & $3 \%$ & $22 \%$ & $8 \%=$ Posgrado \\
$61-70$ & $0.2 \%$ & $10.8 \%$ & \\
\hline
\end{tabular}

En la caracterización de los huertos se encontró que en su mayoría (79\%) son urbanos (Tabla 2), el 50\% huertos mixtos (cultivan tanto en suelo como en macetas o contenedores), probablemente a cusa de que el espacio es mapas reducido en las zonas urbanas (Erazo, 2012). En cuanto al tiempo dedicado al huerto, el $67 \%$ de las personas le asigna menos de 6 horas a la semana y el $33 \%$ dedica más de 12 horas a la semana. El porcentaje de uso de bitácora por los productores es del $25 \%$, a pesar de que el $89 \%$ considera importante emplearla para el registro de las actividades en el huerto, y la consideran como una ventaja para mejorar los procesos de planeación y análisis del sistema agrícola (Cardozo, 2013).

Tabla 2. Características de los huertos de la RAUPX.

\begin{tabular}{cccccc}
\hline \multicolumn{3}{c}{ Cantidad de huertos } & \multicolumn{3}{c}{ Tipo de huerto } \\
Huerto & Cantidad & Porcentaje & Huerto & Cantidad & Porcentaje \\
\hline Urbano & 22 & 79 & *Blando & 6 & 17 \\
Periurbano & 6 & 21 & $* *$ Duro & 8 & 33 \\
Total & 28 & 100 & ***Mixto & 14 & 50 \\
& & & Total & 28 & 100 \\
\hline
\end{tabular}

*Siembra directa en suelo. ** Siembra en contenedor *** Siembra directa en suelo y contenedores.

Las especies hortícolas más frecuentes pertenecen a las familias: Brassicaceae, Fabácea y Solanácea con seis especies cada una; y en menor cantidad se encontraron especies condimentarias, medicinales y hortícolas de las familias Amaranthaceae, Amaryllidaceae y
Lamiaceae, como se observa en la tabla 3. La ventaja de contar con un número elevado de especies vegetales ayuda a diseñar y programar la rotación y asociación de cultivos, así como a las prácticas de manejo para el control de plagas (Nicholls, 2008). 
Tabla 3. Diversidad de especies medicinales, condimentarias y hortalizas en huertos.

\begin{tabular}{|c|c|c|c|c|c|}
\hline Familia & Especie & $\begin{array}{l}\text { Nombre } \\
\text { común }\end{array}$ & Familia & Especie & $\begin{array}{l}\text { Nombre } \\
\text { común }\end{array}$ \\
\hline \multirow[t]{2}{*}{$\begin{array}{c}\text { A } \\
\text { Amaranthaceae }\end{array}$} & Amaranthus sp. & Quelite & $\begin{array}{c}\mathbf{E} \\
\text { Euphorbiaceae }\end{array}$ & $\begin{array}{l}\text { Cnidoscolus } \\
\text { aconitifolius }\end{array}$ & Chaya \\
\hline & Salvia hispanica & Chía & $\begin{array}{c}\mathbf{F} \\
\text { Fabaceae }\end{array}$ & Arachi shypogaea & Cacahuate \\
\hline \multirow[t]{3}{*}{ Alliaceae } & Allium glandulosum & Cebollín & & Medicago sativa & Alfalfa \\
\hline & $\begin{array}{c}\text { Allium } \\
\text { ampeloprasum }\end{array}$ & Poro & & Pachyrhizu serosus & Jícama \\
\hline & Allium cepa & Cebolla & & Phaseolus coccineus & Acoyote \\
\hline \multirow[t]{4}{*}{ Asteraceae } & Calendula officinalis & Caléndula & & Phaseolus vulgaris & Frijol \\
\hline & $\begin{array}{c}\text { Artemisia } \\
\text { absinthium }\end{array}$ & Ajenjo & & Vicia faba & Haba \\
\hline & Lactuca sativa & Lechuga & $\begin{array}{c}\mathbf{L} \\
\text { Lauraceae }\end{array}$ & Cinnamomum verum & Canela \\
\hline & $\begin{array}{l}\text { Matricaria } \\
\text { chamomilla }\end{array}$ & Manzanilla & Lamiaceae & Origanum vulgare & Orégano \\
\hline Asphodelaceae & Aloe vera & Sábila & & Thymus vulgaris & Tomillo \\
\hline \multirow[t]{4}{*}{ Apiaceae } & Coriandrum sp. & Cilantro & & Mentha piperita & Menta \\
\hline & Daucus carota & Zanahoria & & Mentha spicata & Hierbabuena \\
\hline & $\begin{array}{c}\text { Petroselinum } \\
\text { crispum }\end{array}$ & Perejil & & Melissa offinalis & Toronjil \\
\hline & Apium graveolens & Apio & & Plectranthus oloroso & Vaporub \\
\hline \multirow[t]{6}{*}{$\begin{array}{c}\text { B } \\
\text { Brassicaceae } \\
\end{array}$} & Brassica olearaceae & Brócoli & $\begin{array}{c}\mathbf{M} \\
\text { Malvaceae }\end{array}$ & Hibiscus sabdariffa & Jamaica \\
\hline & Brassica rapa & Mostaza & $\begin{array}{c}\mathbf{N} \\
\text { Nyctaginaceae }\end{array}$ & Bougainvillea glabra & Bugambilia \\
\hline & Eruca vesicaria & Arugula & $\begin{array}{c}\mathbf{O} \\
\text { Orchidaceae } \\
\end{array}$ & Vanilla planifolia & Vainilla \\
\hline & Raphanus sativus & Rábano & $\begin{array}{c}\mathbf{P} \\
\text { Piperaceae } \\
\end{array}$ & Piper nigrum & Pimienta \\
\hline & Lepidium sativum & Berros & Poaceae & Zea mays & Maíz \\
\hline & Brassica alba & Nabo & & Cymbopogon citratus & $\begin{array}{l}\text { Zacate } \\
\text { limón }\end{array}$ \\
\hline $\begin{array}{c}\mathbf{C} \\
\text { Cactaceae } \\
\end{array}$ & Opuntia crassa & Nopal & Polygonaceae & Rumexa ceto & $\begin{array}{l}\text { Lengua de } \\
\text { vaca }\end{array}$ \\
\hline \multirow[t]{4}{*}{ Chenopodiaceae } & Beta vulgaris & Acelga & Portulacaceae & Portulaca oleracea & Verdolaga \\
\hline & $\begin{array}{c}\text { Dysphania } \\
\text { ambrosioides }\end{array}$ & Epazote & $\begin{array}{c}\mathbf{T} \\
\text { Tropaeolaceae }\end{array}$ & Tropaeolum majus & Mastuerzo \\
\hline & Spinaciaoleracea & Espinaca & $\begin{array}{c}\mathbf{Z} \\
\text { Zingiberaceae }\end{array}$ & Zingiber officinale & Jengibre \\
\hline & $\begin{array}{c}\text { Chenopodium } \\
\text { bonnus }\end{array}$ & Huazontle & & & \\
\hline \multirow[t]{2}{*}{ Cucurbitaceae } & Sechium edule & Chayote & & & \\
\hline & Cucurbita maxima & Calabaza & & & \\
\hline
\end{tabular}


Como resultado del concepto de AUyP, plaga y su control (Tabla 4), los productores presentan un sesgo en 3 de las 5 preguntas (ligeras diferencias de los conceptos), lo cual se atribuye a que omiten o desconocen la relación de las actividades relacionadas con la agricultura urbana y esto puede deberse al nivel escolar y empírica (Matienzo et al., 2008).

Tabla 4. Percepción de productores sobre temas de agricultura urbana y periurbana.

\begin{tabular}{cc}
\hline Pregunta & *Valor en escala Likert \\
\hline Concepto de Agricultura Urbana y & 2.07 \\
Periurbana(AUyP) & \\
Como ayuda la AUyP al ambiente & 1.6 \\
Concepto de plaga & 3 \\
Importancia de plagas & 3.96 \\
Uso y valor de bitácora & 4.29 \\
\hline *1 Muy bajo, 2 Bajo, 3 Regular, 4 Alto, 5 Muy alto
\end{tabular}

Las prácticas fitosanitarias se organizaron conforme a la complejidad de actividades, que realizan de manera preventiva para el control de plagas y manejo de la huerta, las cuales son básicascomo actividades de reciclar material para sembrar y las más complejas como la compra de organismos entomófagos para el control biológico (Tabla 5). En relación con el nivel de conocimientos y los juicios personales sobre los problemas que enfrentan y de los insumos necesarios para su realización estas prácticas denotan la amplia experiencia de los productores, que van adquiriendo mayor complejidad a través del tiempo (Soto et al., 2010; Nolasco et al., 2016).

De acuerdo con el análisis de tendencias centrales, se observó que existen huertos con superficies amplias de $500 \mathrm{~m}^{2}$ y pequeñas de $4 \mathrm{~m}^{2}$, esto influye de manera directa en el número de plagas, puesto que se ha reportado que en pequeñas áreas se mantienen prácticas usando el menor esfuerzo (Cantor, 2010). En cuanto a la implementación de estas variables en relación con el supuesto de que una alta biodiversidad favorece la conservación de organismos benéficos y la resistencia del agrosistema ante ataques potenciales de plagas mono y polífagas (Nicholls, 2008), se observó que con el aumento de la diversidad la incidencia de plagas fue mayor, lo cual indica que las practicas preventivas no son adecuadas ni suficientes (Rodríguez y Companioni, 2006). 
Tabla 5. Complejidad de las prácticas fitosanitarias en los huertos urbanos.

\begin{tabular}{|c|c|c|c|}
\hline Categoría & Tipo de control & Actividad / Manejo / Técnica/ & Frecuencia \\
\hline \multirow{6}{*}{$\begin{array}{l}\text { Básica } \\
\text { Simple }\end{array}$} & \multirow{6}{*}{$\begin{array}{l}\text { Cultural, Mecánico, } \\
\text { Físico }\end{array}$} & Reutilización de materiales (PET, llantas) & 28 \\
\hline & & Deshierbe/poda & 26 \\
\hline & & Abonos & 21 \\
\hline & & Riego y captación de agua & 11 \\
\hline & & Arropes & 6 \\
\hline & & Eliminación manual de insectos & 17 \\
\hline \multirow{6}{*}{$\begin{array}{c}\text { Media } \\
\text { Compleja }\end{array}$} & \multirow{6}{*}{$\begin{array}{c}\text { Etológico, Biológico } \\
\text { por conservación }\end{array}$} & Composta / Manejo de residuos & 16 \\
\hline & & Rotación de familias & 12 \\
\hline & & Asociación de familias & 12 \\
\hline & & $\begin{array}{l}\text { Favorecer organismos benéficos } \\
\text { /depredadores }\end{array}$ & 11 \\
\hline & & Bioplaguicidas/preparados botánicos & 21 \\
\hline & & Manejo etológico & 1 \\
\hline \multirow{4}{*}{$\begin{array}{l}\text { Alta Muy } \\
\text { compleja }\end{array}$} & \multirow{4}{*}{$\begin{array}{l}\text { Genético, Fenológico, } \\
\text { Biológico por } \\
\text { inoculación }\end{array}$} & Calendario de siembra (uso de bitácora) & 8 \\
\hline & & Producción y conservación de semillas & 10 \\
\hline & & $\begin{array}{c}\text { Liberación de organismos benéficos, } \\
\text { estériles y/o depredadores (insectos, hongos } \\
\text { y bacterias). }\end{array}$ & 1 \\
\hline & & $\begin{array}{c}\text { Agricultura biodinámica (uso de las fases } \\
\text { lunares y fenológicas). }\end{array}$ & 2 \\
\hline
\end{tabular}

De acuerdo con el análisis de tendencias centrales, se observó que existen huertos con superficies amplias de $500 \mathrm{~m}^{2}$ y pequeñas de $4 \mathrm{~m}^{2}$, esto influye de manera directa en el número de plagas, puesto que se ha reportado que en pequeñas áreas se mantienen prácticas usando el menor esfuerzo (Cantor, 2010). En cuanto a la implementación de estas variables en relación con el supuesto de que una alta biodiversidad favorece la conservación de organismos benéficos y la resistencia del agrosistema ante ataques potenciales de plagas mono y polífagas (Nicholls, 2008), se observó que con el aumento de la diversidad la incidencia de plagas fue mayor, lo cual indica que las practicas preventivas no son adecuadas ni suficientes (Rodríguez y Companioni, 2006). 
Tabla 6. Medidas de tendencia central de las variables de estudio ( $\mathrm{N}=28)$.

\begin{tabular}{ccccc}
\hline & Prácticas & $\begin{array}{c}\text { Índice de } \\
\text { diversidad de } \\
\text { Shannon }\end{array}$ & Artropofauna & $\begin{array}{c}\text { Tamaño del huerto } \\
\left(\mathrm{m}^{2}\right)\end{array}$ \\
\hline Media & 7.25 & 3.15 & 7.68 & 88.25 \\
Mediana & 6.00 & 3.13 & 7.00 & 25.00 \\
Desviación estándar & 2.78 & 0.38 & 3.24 & 141.70 \\
Varianza & 7.75 & 0.15 & 10.52 & $2,0081.01$ \\
Min & 4 & 2.52 & 3 & 4 \\
Max & 14 & 3.81 & 16 & 500 \\
\hline
\end{tabular}

\section{CONCLUSIONES}

La mayoría de los productores de la Red de Agricultura Urbana y Periurbana de Xalapa tienen huertos situados en zonas urbanas. En cuanto a la participación de género, predominaron las mujeres. Las especies que se cultivan con más frecuencia pertenecen a las familias Amaranthaceae, Amaryllidaceae, Lamiaceae, Brassicaceae, Fabaceae y Solanaceae. Por otro, lado los conceptos de plaga y AUyP presentaron diferencias entre los productores, dada la percepción de cada uno. Las prácticas que realizan con mayor frecuencia por su complejidad son básicas-simples, con baja periodicidad en rotación y asociación de cultivos. Así mismo, el tamaño de huerto e índice de diversidad de Shannon están moderadamente relacionados con la incidencia de plagas, lo cual hace que el productor realice acciones preventivas.

\section{LITERATURA CITADA}

Altieri, M., Companioni, N., Cañizares, K., Murphy, C., Rosset, P., Bourque, M. y Nicholls, C. 2000.
The greening of the "barrios": urban agriculture for food security in Cuba. Agriculture and Human Values 16(2): 131-140. https://doi.org/10.1023/A:100754 5304561

Cantor, K. M. 2010. Agricultura urbana: Elementos valorativos sobre su sostenibilidad. Cuadernos de Desarrollo Rural 7(65): 59-84.

Cardozo, E. G. 2013. Sistema para la elaboración de huertas urbanas como autoabastecimiento alimenticio en los hogares vulnerables. 133(8): 82.

Companioni, N., Ojeda, Y. y Páez, E. 2001. La agricultura urbana en Cuba. 99-112.

Escalona, M., Cortes, Z. M., Isabel, M. y Armella, N. 2016. Xalapa City urban and peri urban agriculture network, self-managed experience that creates community and citizenship. 4-14 p.

Erazo, N. 2012. Agricultura Urbana como alternativa de planificación sostenible del medio ambiente urbano de la ciudad de Loja. Ecuador. 255 p. 
Matienzo, Y., Elizondo, A. I., Veitía, M., Botta, E., Grana, Y., Carmenate, H. y Matamoros, M. 2008. Percepción de los agricultores sobre las prácticas que contribuyen a la conservación de artrópodos biorreguladores de plagas en la agricultura urbana de Ciudad de La Habana. Agricultura Orgánica 14(2): 3739.

Nicholls, C. 2008. Control biológico de insectos: un enfoque agroecológico. Ciencia y Tecnología Universidad de Antioquia. Colombia 65-66 p.

Nolasco, B., Méndez, J., Enríquez, F. y Berdeja, R. 2016. Caracterización agroecológica de los sistemas de traspatio del municipio de Caxhuacan, Puebla. Revista Biológico Agropecuaria Tuxpan 6(1).

Rivera, A. 2006. Hábitos alimentarios en estudiantes de la Universidad
Juárez Autónoma de Tabasco. Revista Cubana de Salud Pública 32(3): 10-20.

Rodríguez, N. y Companioni, N. 2006. Situación actual, perspectivas y retos de la Agricultura urbana en Cuba. Revista Agricultura Orgánica 4 (12): 2.

Soto, G., Carral, G. T. y Huerta, J. M. 2010. Percepciones sobre la relación ser humano- naturaleza y sobre los modelos agrícolas. Extensión Agrícola de la Universidad de Puerto Rico 9(54): 35.

Toledo, V. 2002. Agroecología, sustentabilidad y reforma agraria: la superioridad de la pequeña producción familiar. Agroecología y Desenvolvimiento Rural Sustentable 3(2): 27-36.

Verdugo, M. y Sabeh, N. 2002. Evaluation of perception of quality of life in childhood. Psicothema 14(1): 86-91. https://doi.org/10.1136/adc.86.1.14

Copyright (c) 2018 S aúl Ortiz Riveros, Nancy Dom inguez González, Noel Reyes Pérez, Marycruz Abato Zárate, Carlos R. Cerdán Cabrera, Miguel A Escalona Aguilar y J. Armando L ozada G arcia

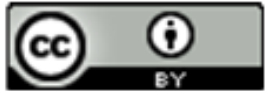

Este tex to está protegido por una licencia Creative Commons 4.0

\footnotetext{
Usted es libre para Compartir —copiar y redistribuir el $\mathrm{m}$ aterial en cualquier medio o formato- $\mathrm{y}$ Adaptar el documento —remezclar, transformar y crear a partir del material- para cualquier propósito, incluso para fines com erciales, siempre que cumpla la condición de:

Atribución: Usted debe dar crédito a la obra original de manera adecuada, proporcionar un enlace a la licencia, e indicar si se han realizado cambios. Puede hacerlo en cualquier forma razonable, pero no de forma tal que sugiera que tiene el apoyo del licenciante o lo recibe por el uso que hace de la obra.

Resumendelicencia - Textocompletodelalicencia
} 